\title{
Traumatisme isolé du pancréas après une rixe
}

\section{Isolated Injury to the Pancreas after Abdominal Trauma}

\section{E. Hansconrad $\cdot$ P. Plaisance}

Reçu le 11 juin 2018 ; accepté le 30 juillet 2018

(C) SFMU et Lavoisier SAS 2018

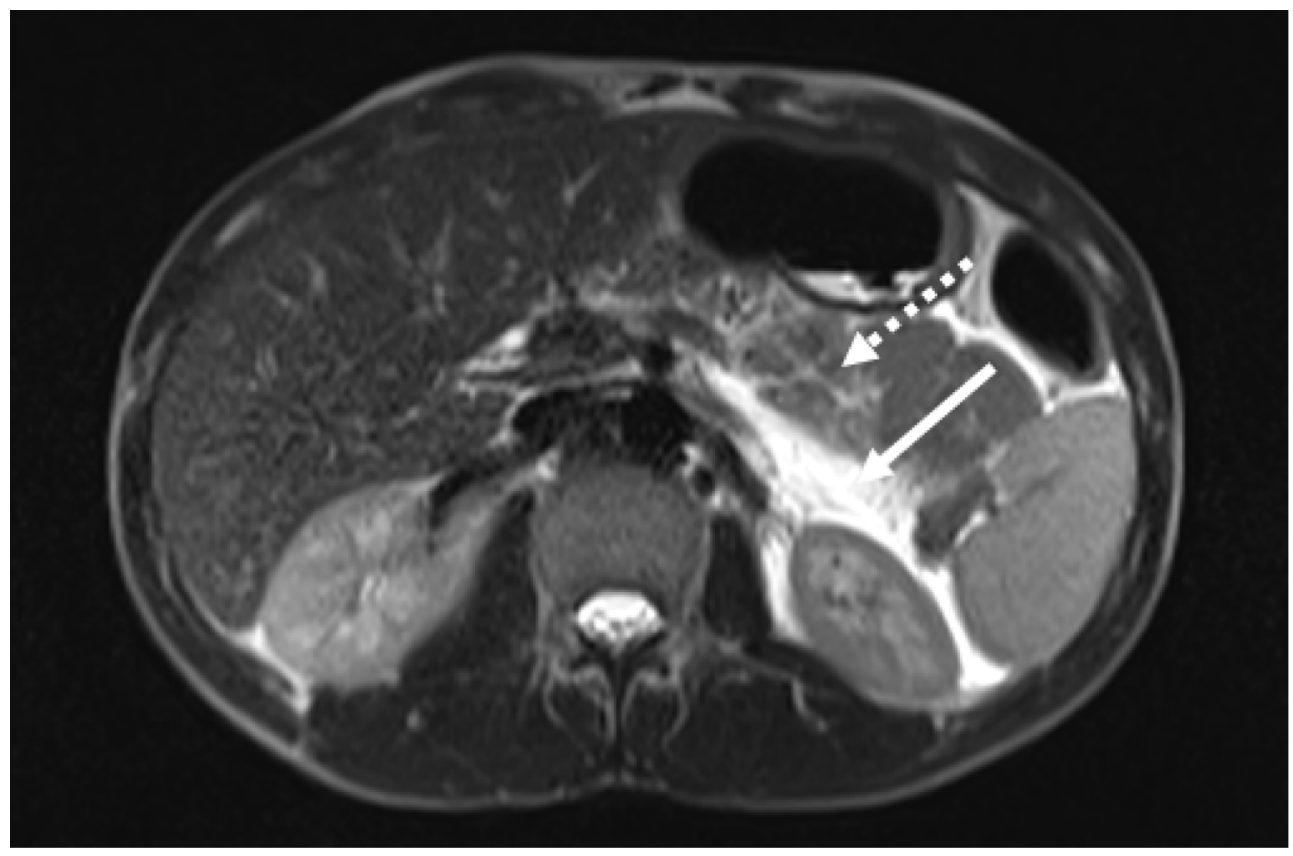

Fig. 1 IRM abdominale en séquence T2 montrant un aspect hétérogène en hypersignal du corps et de la queue du pancréas traduisant une contusion (flèche pointillée) avec un épanchement intrapéritonéal associé (flèche pleine)

Un homme de 23 ans est amené par les pompiers pour un traumatisme abdominal isolé avec une douleur prédominant en épigastrique mais sans nausées ni vomissements. Le patient est stable sur le plan hémodynamique avec une tachycardie isolée à 118/minute dans un contexte algique (EVA à $7 / 10)$ nécessitant une titration morphinique. Un Hémoccue ${ }^{\circledR}$

\footnotetext{
E. Hansconrad $(\varangle) \cdot$ P. Plaisance

Service d'accueil des urgences, hôpital Lariboisière,

Assistance publique-Hôpitaux de Paris (AP-HP),

2, rue Ambroise-Paré, F-75010 Paris, France

e-mail : erwin.hansconrad@aphp.fr

\section{P. Plaisance}

Faculté de médecine Lariboisière-Saint-Louis, université Paris-Diderot-Paris-VII,

10, avenue de Verdun, F-75010 Paris, France
}

est réalisé à 14,6 g/dl. L'examen clinique révèle une défense à la palpation de l'épigastre. Le reste de l'abdomen est sensible, sans défense ni contracture. L'examen cardiopulmonaire est normal. Le bilan biologique montre une hyperlipasémie à $488 \mathrm{UI} / 1$ (6 N), le bilan hépatique et la fonction rénale sont normaux. Une TDM abdominale réalisée en urgence fait suspecter une lésion du corps du pancréas évocatrice de fracture du pancréas associé à un épanchement intrapéritonéal. Une IRM abdominale (Fig. 1) de complément conclut à une contusion du corps et de la queue du pancréas sur 30 à $40 \%$ du parenchyme classée type III selon la classification de l'American Association for the Surgery of Trauma (AAST). La décision d'une exploration chirurgicale a été prise. Il n'a pas été constaté de fracture ni de lésion du canal de wirsung du pancréas. Un drainage simple au contact du pancréas a été laissé en place. 
Les traumatismes du pancréas sont rares $(0,2$ à $6 \%$ de l'ensemble des traumatismes de l'abdomen [1]) et fréquemment associés à d'autres lésions, en particulier duodénales. Le traumatisme isolé du pancréas est de diagnostic souvent difficile de par son tableau clinique initial extrêmement variable. Certains sont méconnus et ne sont découverts que tardivement au stade de complications [2]. La lipasémie peut être utile lors de l'exploration d'une douleur épigastrique posttraumatique. Cependant, elle est peu sensible et peu spécifique et est augmentée dans seulement $50 \%$ des cas des traumatismes pancréatiques. La TDM abdominale est l'examen de référence qui permet de faire le diagnostic de lésion pancréatique et de classifier la sévérité des lésions. Néanmoins, elle détecte une anomalie du canal pancréatique principal dans seulement $50 \%$ des cas. La cholangiopancréato-IRM en complément permet une meilleure visibilité pour la recherche de rupture du canal pancréatique principal. Le traitement des traumatismes pancréatiques dépend essentiellement de l'intégrité ou non du canal pancréatique principal [2]. On privilé- giera un traitement médical en l'absence de lésion du canal pancréatique principal et un traitement chirurgical ou endoscopique lors des atteintes canalaires. La mortalité globale chez les patients atteints de traumatismes abdominaux avec atteinte pancréatique varie de 5 à $30 \%$ selon les séries ; cependant, elle n'est directement imputée au pancréas que dans 5 à $10 \%$ de ces décès [1]. La potentielle gravité de ce type de lésion doit nous inciter à réaliser au minimum une TDM abdominale devant tout traumatisme épigastrique suspect à la recherche d'une lésion du pancréas.

\section{Références}

1. Carrel T, Lerut J, Niederhauser U, et al (1990) Diagnosis and treatment of traumatic injuries of the duodenum and pancreas: 21 cases. J Chir 127:438-44

2. Arvieux C, Guillon F, Létoublon Ch, et al (2003) Pancreatic trauma. J Chir 140:261-9 